\title{
Evaluation of Self-Care Status and Its Relationship with Self-Efficacy of Patients with Hypertension
}

\begin{abstract}
Background and Objective: Hypertension is a common disease, often asymptomatic and treatable. Left untreated, this condition often leads to fatal complications. The aim of this study was to investigate the status of self-care and its relationship with self-efficacy of patients with hypertension in Mashhad in 2019.

Materials and Methods: In this descriptive cross-sectional study, 250 patients with hypertension in Mashhad in 1398 were selected by multi-stage method from among patients referred to comprehensive health care centers. Data collection tools were a three-part questionnaire including: demographic information and a standard questionnaire of self-efficacy and self-care of blood pressure. Data were analyzed using t-test, analysis of variance, Mann-Whitney, Kruskal-Wallis, linear correlation and regression by SPSS software version 20 with a significance level of 0.05 .

Results: Based on the findings, self-efficacy and self-care behaviors with scores of $40.43 \pm 10.13$ and $48.78 \pm 10.66$ were relatively undesirable. There was a significant relationship between self-efficacy and self-care behaviors with job, body mass index, number of sessions of receiving blood pressure counseling, drug use, and history of illness in the father $(p<0.05)$. It is worth mentioning that a positive and significant correlation was observed between the general scores of self-care and self-efficacy $(P=0.001)$ ( $r p=0.82)$.

Conclusion: Due to the significant correlation between self-care behaviors and self-efficacy and because these cases are very effective in preventing and controlling blood pressure. Therefore, it is recommended that educational programs for patients with hypertension to increase adherence to self-care behaviors and improve levels. Patient self-efficacy to be designed and implemented.

Keywords: Hypertension, self-efficacy, self-care behaviors, health promotion, patient Paper Type: Research Article.
\end{abstract}

Citation (Vancouver): Asa Kohnehe Forroudy Z, Gholian-Aval M, Tehrani H, Esmaily H. Evaluation of Self-Care Status and Its Relationship with Self-Efficacy of Patients with Hypertension. Iran J Health Educ Health Promot. Winter 2021;8(4): 336-347. [Persian]x

- Citation (APA): Asa Kohnehe Forroudy Z., Gholian-Aval M., Tehrani H., Esmaily H. Evaluation of Self-Care Status and Its Relationship with Self-Efficacy of Patients with Hypertension. Iranian Journal of Health Education \& Health Promotion., 8(4), 336-347. [Persian]
Zahra Asa Kohnehe Forroudy1,5, MSc in Health Education and Health Promotion, Student Research Committee, Mashhad University of Medical Sciences, Iran.

Research Center for Social Factors Affecting Health, Mashhad University of Medical Sciences, Iran

Mahdi Gholian-Aval

* Associate Professor, Department of Health Education and Health Promotion, School of Health, Mashhad University of Medical Sciences, Iran. (corresponding author) gholianam@mums.ac.ir

Research Center for Social Factors Affecting Health, Mashhad University of Medical Sciences, Iran

Hadi Tehrani

Assistant Professor, Department of Health Education and Health Promotion, School of Health, Mashhad University of Medical Sciences, Iran.

Research Center for Social Factors Affecting Health, Mashhad University of Medical Sciences, Iran

Habibollah Esmaily

Professor, Department of Epidemiology and Biostatistics, School of Public Health, Mashhad University of Medical Sciences, Iran.

Research Center for Social Factors Affecting Health, Mashhad University of Medical Sciences, Iran

Received: 2020/06/7

Accepted: 2020/09/29

Doi: 10.29252/ijhehp.8.4.1 


\section{بررسى وضعيت خودمراقبتى و ارتباط آن باخودكارآمدى بيماران مبتلا به فشارخون بالا}

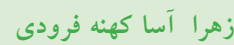

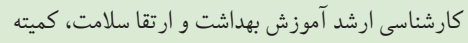

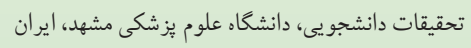

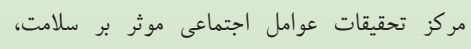
دانشكاء علوم بز شكى مشهد، ايران مهدى قليان اول

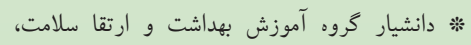

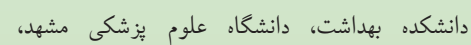
gholianam@mums.ac.ir

ايران(نو يسنده مسئول)

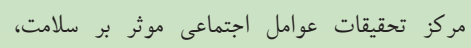
دانشكاء علوم يزشكى مشهد، ايران

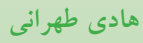

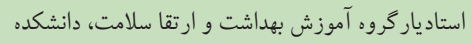

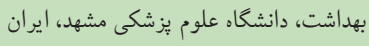

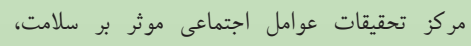
دانشكاء علوم بِّشكى مشهد، ايران حبيب الله اسماعيلى

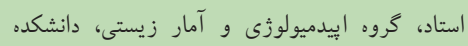

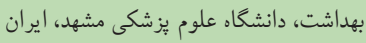

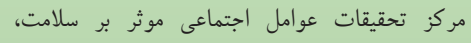

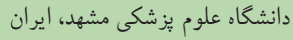

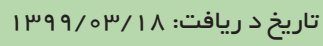
تاريخ يذيرش:

\section{ن}

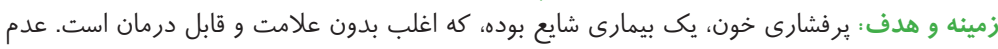

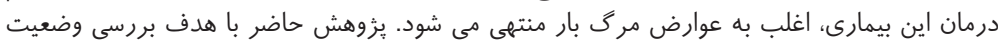

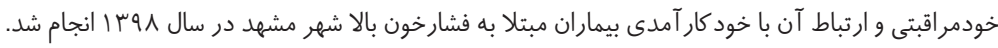

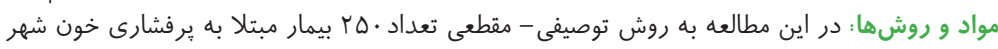

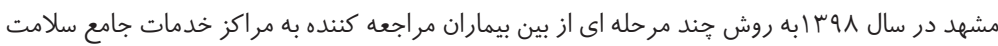

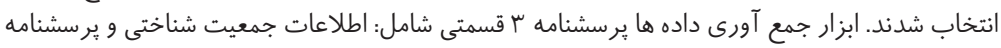

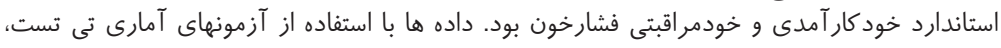

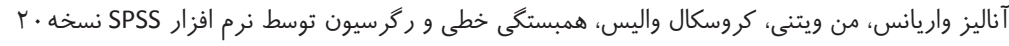

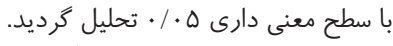

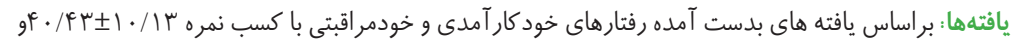

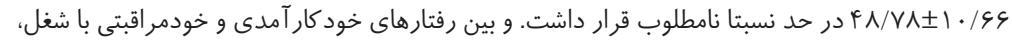

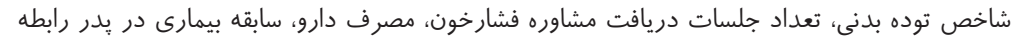

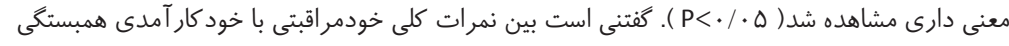

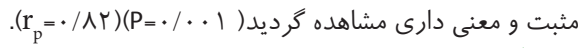

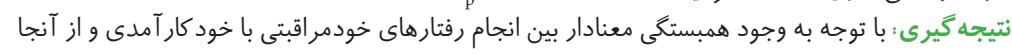

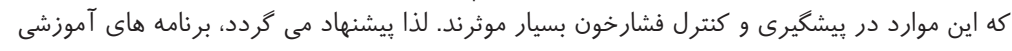

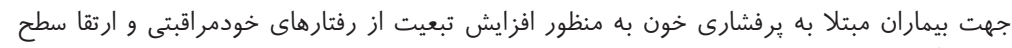

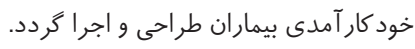

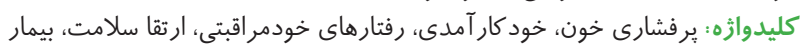
نوع مقاله : مطالعه يزوهشى.

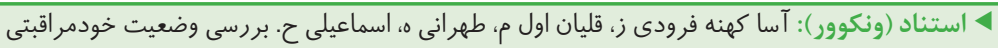

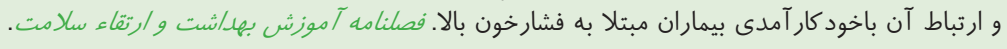

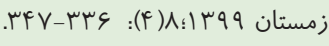

4 استناد (APA): آسا كهنه فرودى، زهر ا؛ قليان اول، مهدى؛ طهر انى، هادى؛ اسماعيلى، حبيب الله. (زمستان

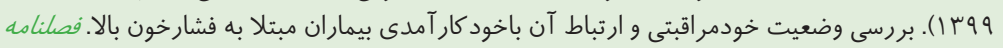

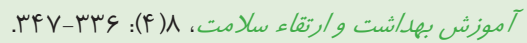




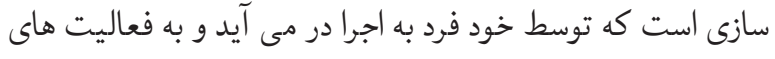

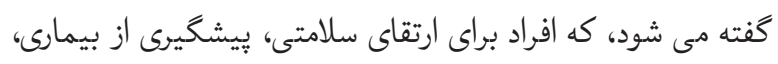

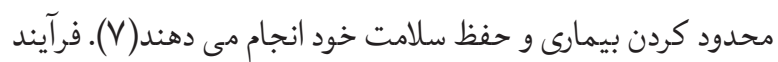

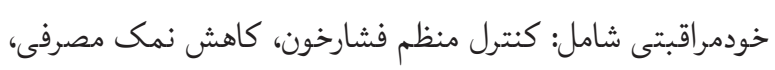

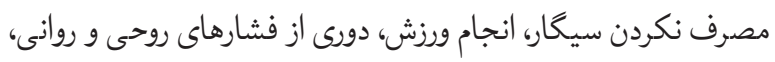

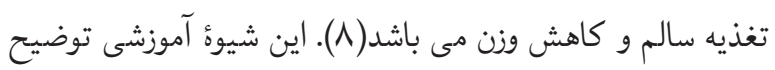

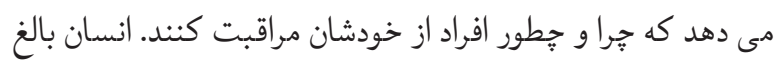

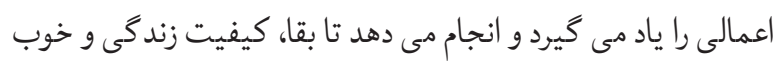

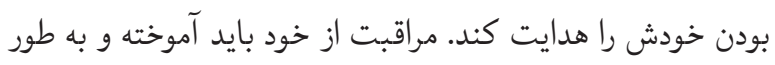

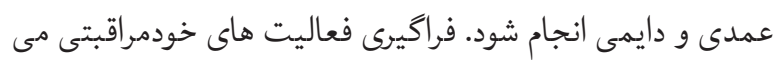

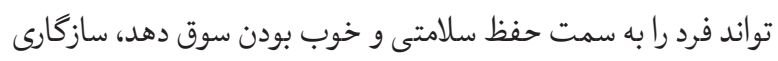

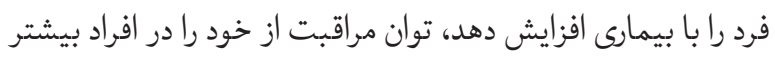

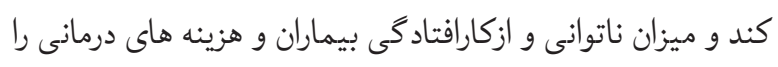

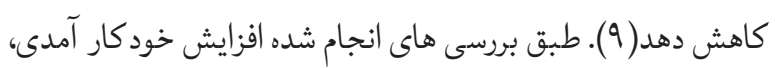

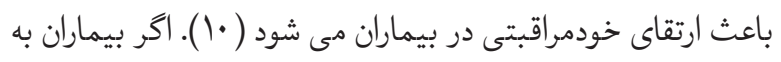
تواناييشان در تغيير و ارتقاى وضعيت سلاتنشان از طريق مشاركت

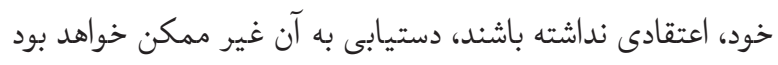

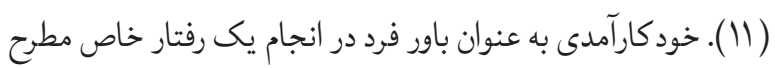

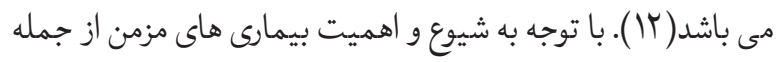

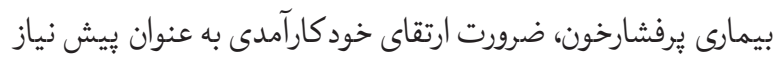

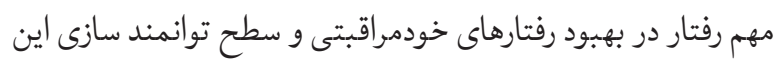

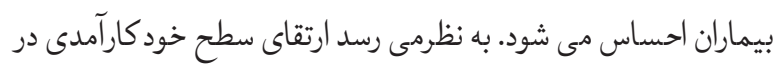

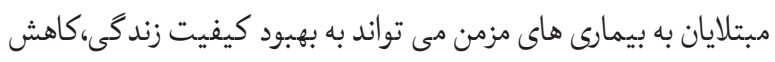

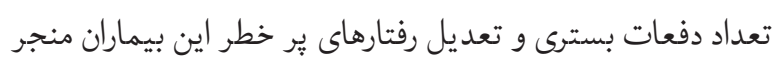

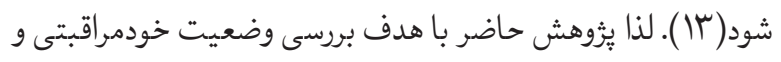

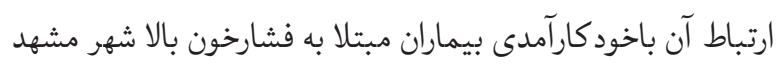
در سال גar| انجام بذيرفت.

$$
\text { مو ادو ورشها }
$$
اين مطالعه توصيفى - مقطعى بر روى تمام بيماران مبتلا به فشارخون
در قرن آميلادى، برجستهترين واقعهاى كه جوامع با آن مواجهاند، شيوع

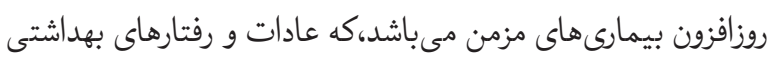

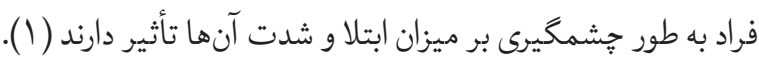

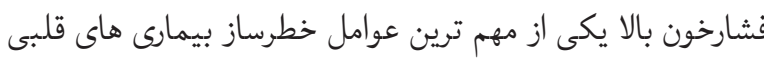

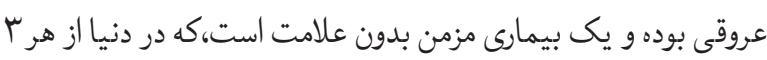

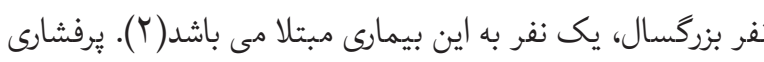

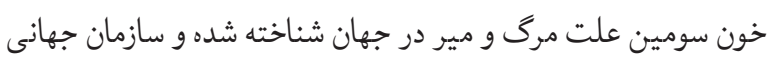
بهداشت WHO نيز سال سا. r را به عنوان مبارزه با بيمارى فشار

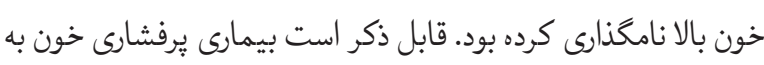

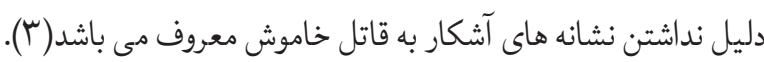

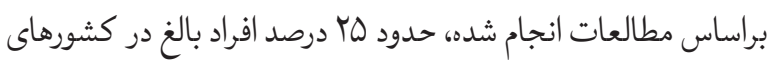
مختلف به بيمارى برفشارى خون مبتلا هستند كه با افزايش سن،

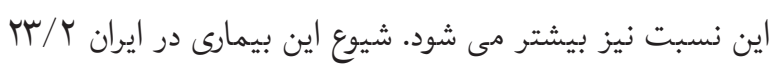

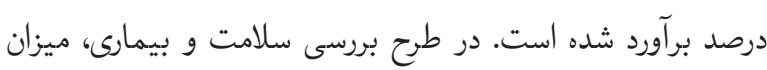

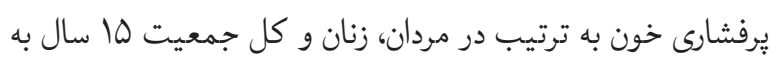

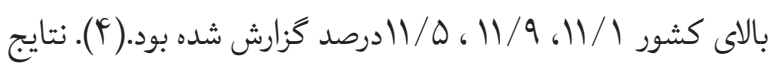

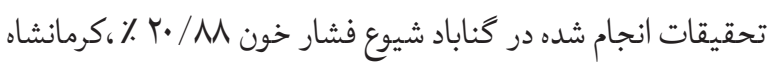

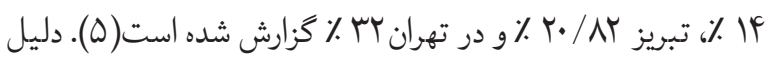

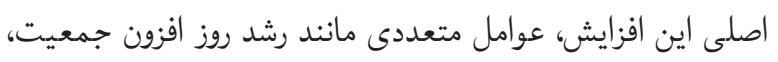

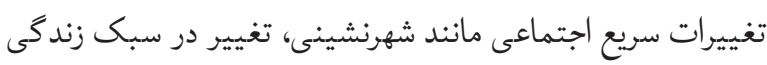

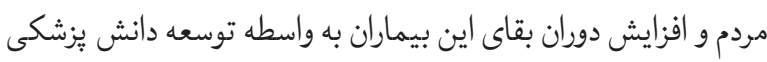

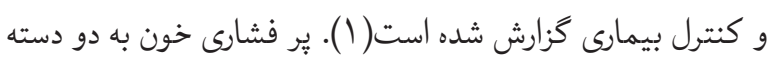

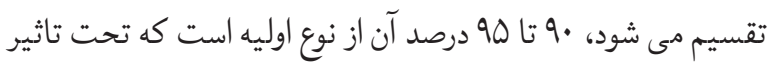
عواملى مانند عوامل زنتيكى، فيزيولوزيك، عوامل خطر قابل نابل اصلاح مانند هاقى، افزايش نمك مصرفى، الكل، سبك زندگى كم تحرك،

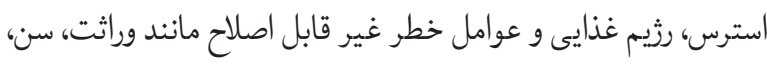

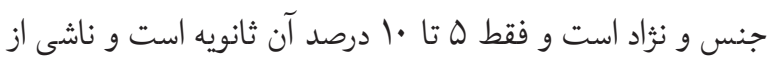
اختلال هاى كليوى، عروقى، غدد درون رين، عصب شناختى، حاملكى نانى و واكنش به داروهاست(9). بديهى است كنترل فشار خون به درجيه

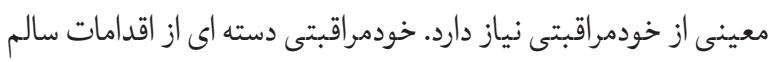


در اين مطالعه، جمع آورى اطلاعات با استفاده از يرسشنامه سه قسمتى صورت كرفت. اين برسشنامه به روش مصاحبه با بيماران

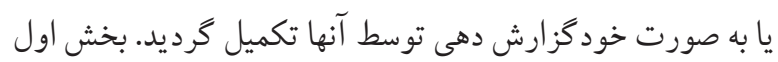
اطلاعات جمعيت شناختى شامل سن، جنس، شغل، سطح تحصيلات،

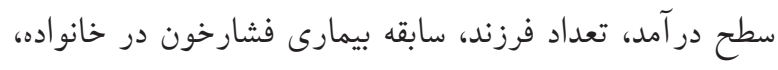

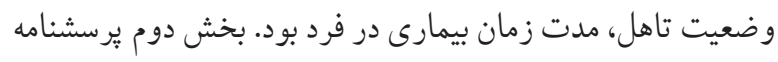

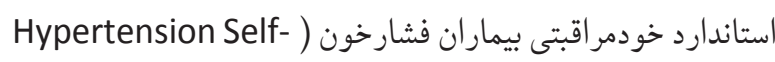
بود كه مشتمل بر · Care Profile) HTN-SCP

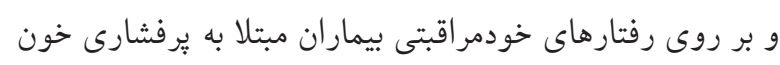

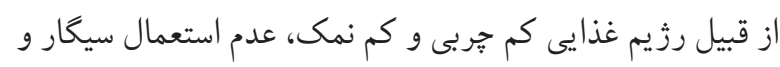

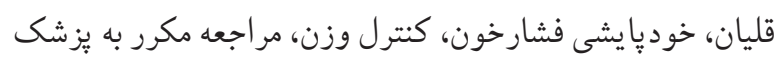

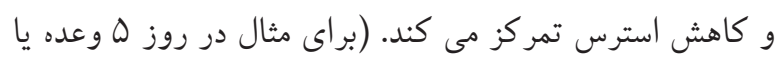

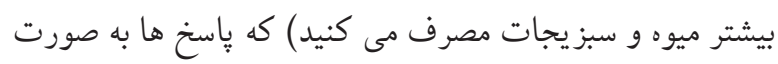

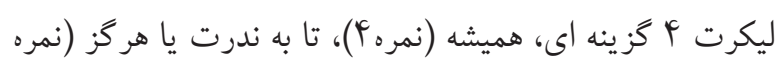

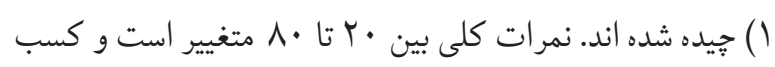

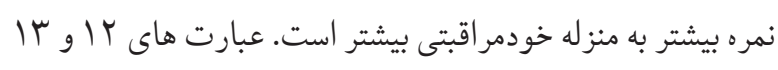

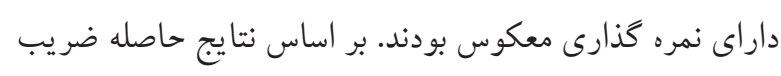

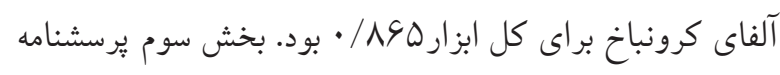

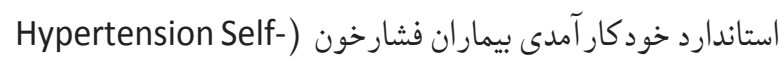
Care Profile) HTN-SCP

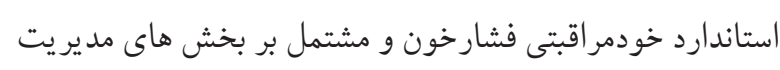

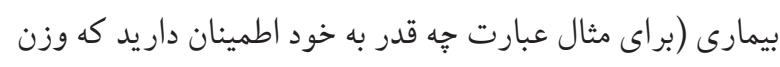

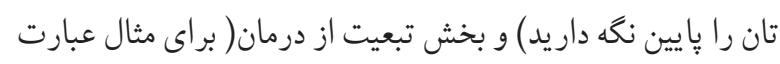

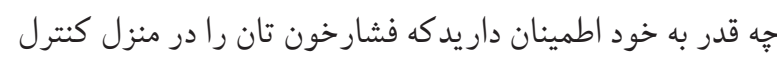

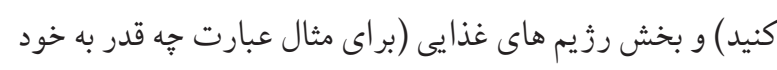

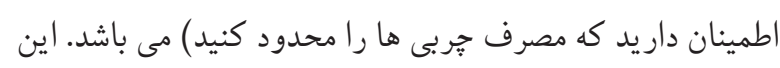
ابزار داراى IV عبارت است كه باسخ هاى آن در مقياس ليكرت جهار كزينه اى كاملا مطمئنم (نمره Y)، تا مطمئن نيستم ( نمرها )

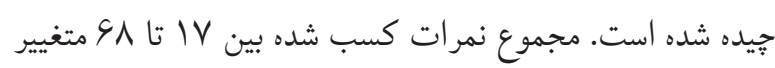

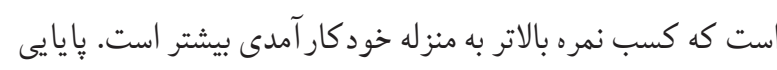

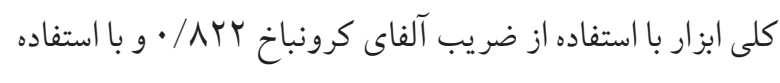

بالا شهر مشهد در سال \وس||نجام شد. •ما بيمار مبتلا به فشارخون بالا مراجعه كننده به مراكز خدمات جامع سلامت شهر مشهد در

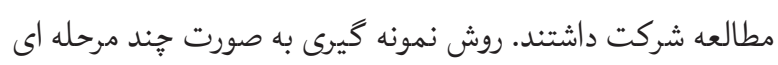

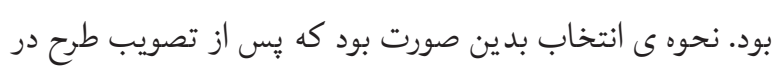

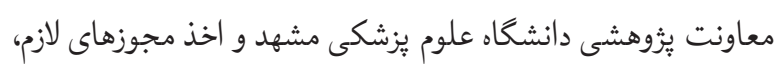

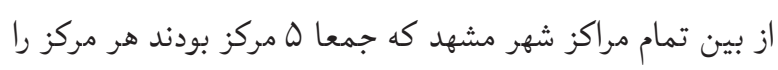
به صورت خوشه مجزا در نظر كرفته و به صورت تصادفى و با قرعه منه

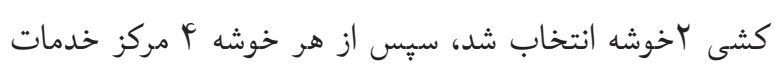
جامع سلامت به صورت تصادفى و با قرعه كشى انتخاب كرديد. با مراجعه به مراكز خدمات جامع سلامت بركزيده از بين بيماران مبتلا

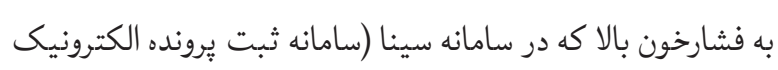

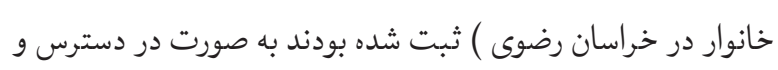
به نسبت جمعيت تحت يوشش هر مركز دعوت به همكارى شد. تبن

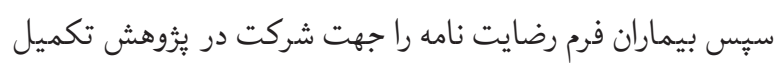

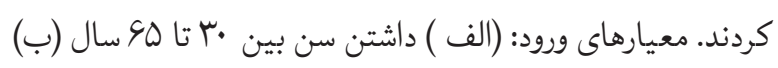

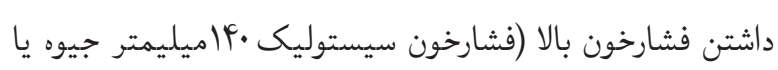

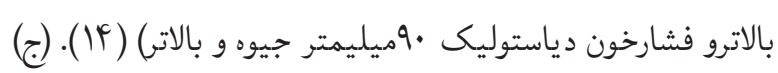

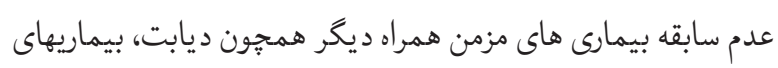

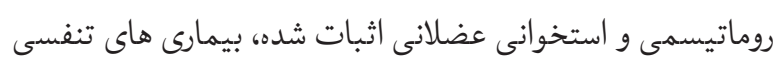
مزمن ( آمفيزم و آسم ) بود. معيار هاى خروج: (الف) عدم تمايل شخصى به ادامه همكارى (ب) سكته هاى مغزى و قلبى و يا بروز ساير عوارض فشارخون، مرگ به هر دليل بود.

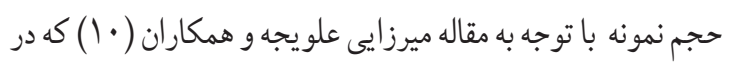

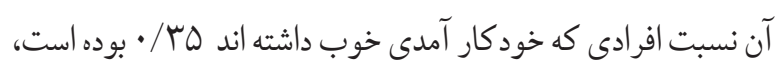

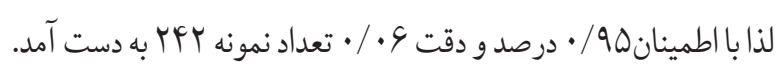

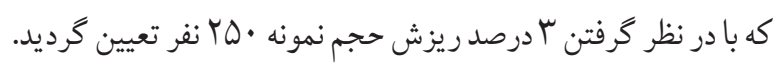
$\mathbf{n}=\frac{\left(z_{1-\alpha / 2}\right)^{2} p(1-p)}{(d)^{2}}$ $\mathrm{n}=\frac{\left(\frac{1}{96}\right)^{2 *} \frac{0}{35\left(1-\frac{0}{35}\right)}}{\left(\frac{0}{\square 6}\right)^{2}}=242+3 \%=250$ 


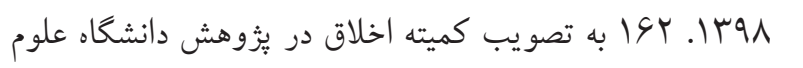

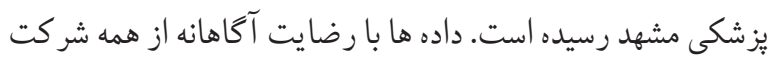

$$
\text { كندكان جمع آورى شده است. }
$$

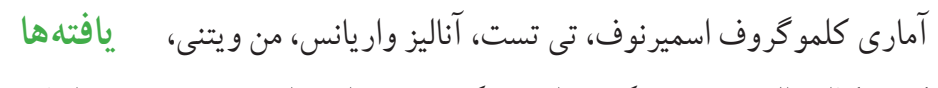

از تست مجدد 90V/ •بود. پِايايى و روايى اين برسشنامه ها در

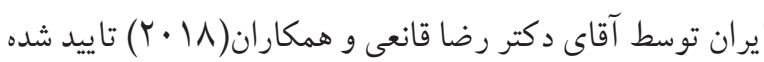

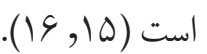
در مطالعه حاضر اطلاعات به دست آمده با استفاده از آزمونهاى

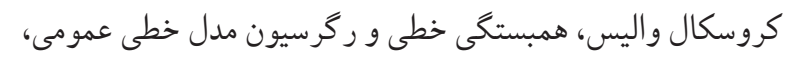

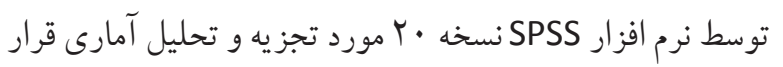

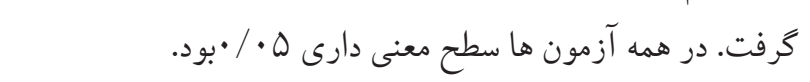

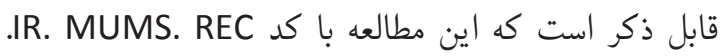
شركت كنند كان در جدول شماره ( (1) ذكر شده است.

بر اساس نتايج مطالعه ميانگین و انحراف معيار سن شركت كنند كان

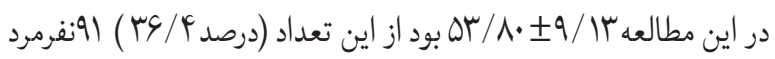

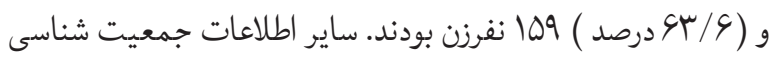
. جدول ا. ارتباط بين متغيرهاى كيفى جمعيت شناختى با خودكار آمدى و خود مراقبتى نمونه هاى مورد بررسى

\begin{tabular}{|c|c|c|c|c|}
\hline (انحراف معيار \)ميانكين & (انحراف معيار ذادميانگين & (درصد) تعداد & سطوح متغير & متغير \\
\hline$F V / q( \pm \mid 1 / F)$ & $\mathrm{rq} / \wedge( \pm 11 / 1)$ & $91($ rs/F) & مرد & \\
\hline$F q / r( \pm 1 \cdot / 1)$ & $\varphi \cdot / V( \pm q / \Delta)$ & $109(\varsigma \mu / \varsigma)$ & زن & بملي \\
\hline $\begin{array}{c}\mathrm{T}=-\cdot / 9 \mathrm{~V} \\
\mathrm{p} \text {-value }=\cdot / \mu r\end{array}$ & $\begin{array}{c}\mathrm{T}=-\cdot / \mathrm{VF} \\
\mathrm{p} \text {-value }=\cdot / \mathrm{F} \Delta\end{array}$ & \multicolumn{3}{|c|}{ نتيجه آزمون تى تست } \\
\hline$r r / G G( \pm r / \cdot \Delta)$ & $\mu r( \pm F / \Delta \Lambda)$ & $\mu(1 / \Gamma)$ & مجرد & \multirow{4}{*}{ ت ت لهل } \\
\hline$F \wedge / \wedge F( \pm \mid 1)$ & $r \cdot / \varsigma q( \pm 1 \cdot / r V)$ & $r \cdot 9(\Lambda \mu / \varsigma)$ & متاهل & \\
\hline$p q / r q( \pm q / \varsigma q)$ & $r \cdot / r \Delta( \pm q / q)$ & $\mu \mid(\mid r / F)$ & بيوه & \\
\hline$F \vee / r \wedge( \pm F / \wedge \Lambda)$ & $r \varsigma / \Delta V\left( \pm q / \Psi^{\mu}\right)$ & $V(Y / \Lambda)$ & مطلقه & \\
\hline $\begin{array}{c}X r=r / 19 * 6 \\
p \text {-value }=\cdot / \Delta r\end{array}$ & $\begin{array}{c}X r=r / I V * * 6 \\
p \text {-value }=\cdot / r \varsigma\end{array}$ & \multicolumn{3}{|c|}{ نتيجه آزمون كروسكال واليس } \\
\hline 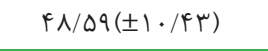 & $r \cdot / \Delta r( \pm q / V)$ & $M \wedge \cdot(V Y / \cdot)$ & شخصى & \multirow{2}{*}{ سكونت } \\
\hline$r q / r \Delta( \pm \mid 1 / r v)$ & $r \cdot / \mid \Delta( \pm \mid 1 / r \Delta)$ & $V \cdot(Y N / \cdot)$ & اجاره & \\
\hline $\begin{array}{c}\mathrm{T}=-\cdot / 4 q \\
\text { p-value }=\cdot / 4 \varphi\end{array}$ & $\begin{array}{c}\mathrm{T}=\cdot /{ }^{\prime} \varsigma \\
\text { p-value }=\cdot / \vee q\end{array}$ & \multicolumn{3}{|c|}{ نتيجه آزمون تى تست } \\
\hline$r q / r \mu( \pm q / q r)$ & $\varphi_{1} / \cdot r( \pm 1 \cdot / . q)$ & $\mid G G(G G / F)$ & دارد & \multirow{2}{*}{ سابقه خانوادگى فشار خون } \\
\hline$F V / q q( \pm \mid l / q \vee)$ & $\mu q / \tau \mu( \pm 1 \cdot / \tau \digamma)$ & $\Lambda F(r \Psi / G)$ & ن & \\
\hline $\begin{array}{c}\mathrm{T}=1 / 1 \Delta \\
\mathrm{p}-\text { value }=\cdot / \mathrm{r} \Delta\end{array}$ & $\begin{array}{c}\mathrm{T}=1 / \mu r \\
\text { p-value }=\cdot / 1 \wedge\end{array}$ & \multicolumn{3}{|c|}{ نتيجه آزمون تى تست } \\
\hline $0 \cdot / V r( \pm 11)$ & $|F| / \varsigma \Delta( \pm 1 \cdot / F \wedge)$ & $\mid r \Delta(\Delta \cdot / \cdot)$ & دارد & \multirow{2}{*}{ سابقه دريافت مشاوره فشار خون } \\
\hline$F G / \Lambda F( \pm q / 9 \vee)$ & $r q / r \cdot( \pm q / q \vee)$ & $\mid r \Delta(\Delta \cdot / \cdot)$ & ن ل & \\
\hline $\begin{array}{c}\mathrm{T}=r / 9 r \\
\text { p-value }=\cdot / \cdots r\end{array}$ & $\begin{array}{c}\mathrm{T}=\mid / 91 \\
\text { p-value }=\cdot / \cdot \Delta\end{array}$ & \multicolumn{3}{|c|}{ نتيجه آزمون تى تست } \\
\hline$\uparrow q / \wedge \varsigma( \pm 1 \cdot / \tau \vee)$ & $\varphi / / . \varphi( \pm q / \Lambda \Lambda)$ & $r \cdot \Lambda(\Lambda \mu / r)$ & دارد & \multirow{2}{*}{ مصرف دارو } \\
\hline$F \Psi / F( \pm 11 / \cdot 1)$ & $\Gamma V / T \wedge( \pm 1 \cdot / 9 r)$ & $\operatorname{Fr}(\mid g / \Lambda)$ & ن & \\
\hline
\end{tabular}


ادامه جدول ا. ارتباط بين متغيرهاى كيفى جمعيت شناختى با خودكارآمدى و خود مراقبتى نمونه هاى مورد بررسى

\begin{tabular}{|c|c|c|c|c|}
\hline $\begin{array}{c}\mathrm{T}=\mu / \Delta \\
\mathrm{p}-\text { value }=\cdot / \cdot \cdot 1\end{array}$ & $\begin{array}{c}\mathrm{T}=r / \cdot r \\
\text { p-value }=\cdot / \cdot r\end{array}$ & \multicolumn{3}{|c|}{ نتيجه آزمون تى تست } \\
\hline$p q / 9( \pm) \cdot / 1 \Delta($ & $F \cdot / 19( \pm q / 9 F($ & $110(\& \varepsilon / \cdot)$ & بلى & \multirow{2}{*}{ فشار خون كنترل شده } \\
\hline $\mathrm{FV} / \wedge \mathrm{r}( \pm) \mathrm{I} / \cdot \mathrm{r}($ & $r \cdot / \cdot r( \pm) \cdot / \mu r($ & $\| r \Delta(\Delta F / \cdot)$ & خير & \\
\hline $\begin{array}{c}\mathrm{T}=1 / \Delta \mathrm{f} \\
\mathrm{p}-\text { value }=\cdot / \mathrm{Ir}\end{array}$ & $\begin{array}{c}\mathrm{T}=\cdot / 49 \\
\mathrm{p}-\text { value }=\cdot / 0\end{array}$ & \multicolumn{3}{|c|}{ نتيجه آزمون تى تست } \\
\hline $01 / 94( \pm) \cdot / G 4($ & $\mathrm{Fr} / \mathrm{s}( \pm q / \Lambda \mathrm{F}($ & $G F(Y \Delta / G)$ & بلى & \multirow{2}{*}{ سابقه بيمارى در يدر } \\
\hline 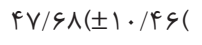 & $r q / r r( \pm) \cdot / \cdot r($ & $\mid \wedge G(V F / F)$ & خير & \\
\hline $\begin{array}{c}\mathrm{T}=r / \mathrm{r} \wedge \\
\text { p-value }=\cdot / \cdot \varphi\end{array}$ & $\begin{array}{c}\mathrm{Z}=-r / \uparrow \varsigma \\
\mathrm{p}-\text { value }=\cdot / \cdot 1\end{array}$ & \multicolumn{3}{|c|}{ نتيجه آزمون تى تست و من ويتنى } \\
\hline$\forall \wedge( \pm q / \Delta S($ & $r q / \Delta F( \pm q / v q($ & $\Lambda \Psi(r / / r)$ & بلى بلى & \multirow{2}{*}{ سابقه بيمارى در مادر } \\
\hline$f q / 19( \pm|1 /| V)$ & $r \cdot / \Lambda V( \pm) \cdot / \mu r($ & $1 \& V(\& \& / \Lambda)$ & خير & \\
\hline $\begin{array}{c}\mathrm{T}=-\cdot / \wedge \mid \\
\mathrm{p}-\text { value }=\cdot / \uparrow \mid\end{array}$ & $\begin{array}{c}\mathrm{Z}=-\cdot / \mathrm{s} \\
\mathrm{p}-\text { value }=\cdot / \mathrm{r}\end{array}$ & \multicolumn{3}{|c|}{ نتيجه آزمون تى تست و من ويتنى } \\
\hline$\uparrow \Psi / \varsigma \wedge( \pm \Lambda / I r($ & $r V / F F( \pm q / \cdot F($ & $r q(11 / 9)$ & آزاد & \multirow{4}{*}{ 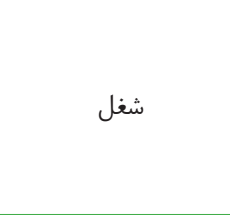 } \\
\hline$\Delta 1 / F \&( \pm) \cdot / r)($ & $\mu F / r( \pm) \cdot / \cdot)($ & $F \Psi(\mid V / r)$ & كارمند و بازنشسته & \\
\hline$F q / r r( \pm) \cdot / . q($ & $r \cdot / \wedge( \pm q / \cdot)($ & $|r|(\Delta r / F)$ & خانه دار & \\
\hline FN/Tr $( \pm \mid r / \cdot 1($ & $r V / G \wedge( \pm) r / \Delta \Delta($ & $\operatorname{FV}(\mid \wedge / \wedge)$ & بيكار & \\
\hline $\begin{array}{c}\mathrm{F}=r / \mu r \\
\mathrm{p}-\text { value }=\cdot / \cdot r\end{array}$ & $\begin{array}{c}\mathrm{F}=\boldsymbol{r} / \mathrm{r} \\
\mathrm{p}-\text { value }=\cdot / \cdot \varphi\end{array}$ & \multicolumn{3}{|c|}{ نتيجه آزمون آناليز واريانس } \\
\hline$\lceil\varsigma / 0)( \pm 9 / 4 \wedge($ & $r q / F \Delta( \pm q / l F($ & $r V(\mid \mathcal{F} / \Lambda)$ & بيسواد & \multirow{3}{*}{ تحصيلات } \\
\hline$\Delta \cdot / \Delta S( \pm) 1 / \Lambda)($ & $\kappa \cdot / \vee s( \pm) 1 / \wedge \Lambda($ & $G F(r \Delta / G)$ & ابتدايى و راهنمايى & \\
\hline$\Psi \wedge / \Delta V( \pm) \cdot / \mu($ & $r \cdot / \Delta \mu( \pm q / \varphi($ & $1 f q(\Delta q / \varepsilon)$ & دييلم و بالاتر & \\
\hline $\begin{array}{c}\mathrm{F}=1 / \mathrm{Vs} \\
\mathrm{p}-\text { value }=\cdot / \mathrm{VV}\end{array}$ & $\begin{array}{c}\mathrm{F}=\cdot / r \mathrm{I} \\
\mathrm{p}-\text { value }=\cdot / \wedge \mid\end{array}$ & \multicolumn{3}{|c|}{ نتيجه آزمون آناليز واريانس } \\
\hline $01 / 94( \pm 11 / 01)$ & $F \mid / F F( \pm 1 \cdot / F F)$ & $\mid \Lambda(V / T)$ & 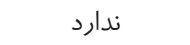 & \multirow{4}{*}{ تعداد فرزند } \\
\hline$\Delta I / r)( \pm) \cdot / r \Delta($ & $F r / q)( \pm) \cdot / r \Delta($ & $r \mu(q / r)$ & يك فرزند & \\
\hline$F q / \mu \xi( \pm) \cdot / \varepsilon \mu($ & $F \cdot / \Delta F( \pm q / G \wedge($ & $V \Gamma(Y q / r)$ & دو فرزند & \\
\hline$F \vee / \varsigma \varphi( \pm) \cdot / \Delta \wedge($ & $r q / s \mathcal{F}( \pm) \cdot / r \wedge($ & $\mid r G(\Delta F / F)$ & سه و بيشتر & \\
\hline $\begin{array}{c}\mathrm{F}=|/ \mathcal{}| \\
\mathrm{p}-\text { value }=\cdot / \tau \mu\end{array}$ & 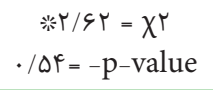 & \multicolumn{3}{|c|}{ 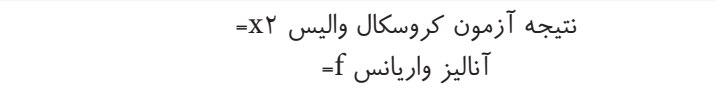 } \\
\hline
\end{tabular}

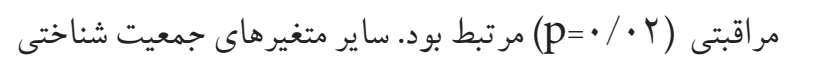
با خود كار آمدى و خود مراقبتى ارتباط معنى دارى نداشتند (جدول

شماره (1)

همجنين بر اساس نتايج يُزوهش خود كار آمدى و خود مراقبتى

ارتباط خطى مستقيمى با يكديخر داشتند( ( + • • (p). تعداد جلسات

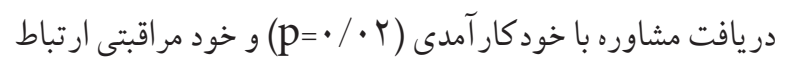

بر اساس نتايج مطالعه سابقه دريافت مشاوره فشارخون با خودمر اقبتى ارتباط معنى دارى داشت ( F • • / = p). مصرف دارو

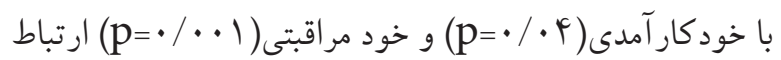
معنى دار داشت. سابقه بيمارى در يدر نيز ارتباط معنى دارى با

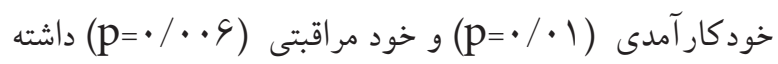

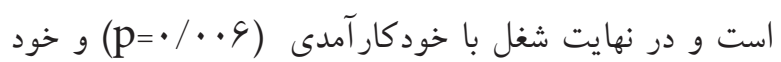




\begin{tabular}{|c|c|c|c|c|c|}
\hline.$/ 9 r$ & .1 .9 & $\cdot / \Lambda \Lambda$ & $-\cdot / \cdot \wedge$ & 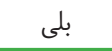 & سابقه بيمارى در \\
\hline- & - & - & ref & خير & ק קנر \\
\hline$\cdot / \cdot r$ & $r / 94$ & $1 / \pi q$ & $-r / l r$ & آزاد & \multirow{4}{*}{ شغل } \\
\hline$\cdot / \cdot r$ & $r / r V$ & $1 / r s$ & $-r / \Lambda \varphi$ & بازنشسته & \\
\hline$\cdot / \cdot r$ & $r / r \Delta$ & $1 / \cdot 1$ & $-Y / Y V$ & خانه دار & \\
\hline- & - & - & ref & بيكار & \\
\hline$\cdot / r F$ & $1 / 19$ & $1 / \cdot r$ & $|/ r|$ & بلى & \multirow{2}{*}{ سابقه دريافت } \\
\hline- & - & - & ref & خير & \\
\hline$\cdot / \cdot r$ & $r / \cdot \Lambda$ & $1 / \cdot 1$ & $r / \mu$ & بلى بلى & \multirow{2}{*}{ مصرف دارو } \\
\hline - & - & - & ref & خير & \\
\hline
\end{tabular}

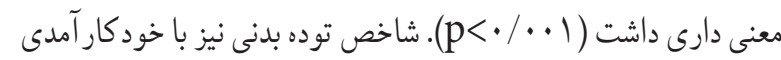

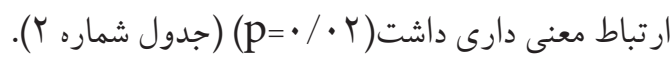

جدل r. ارتباط بين متغيرهاى كمى جمعيت شناختى با خودمراقبتى و خود كارآمدى نمونه هاى مورد بر مسىى

\begin{tabular}{|c|c|c|c|}
\hline خود كار آمدى & خودمراقبتى & (انحراف معيار \) & متغير \\
\hline $\begin{array}{l}\mathrm{r}_{\mathrm{s}}=\cdot / \cdots 1 \\
\mathrm{p}=\cdot / 91 * * 6\end{array}$ & $\begin{array}{l}\mathrm{r}_{\mathrm{s}}=\cdot / \cdot 1 \\
\mathrm{p}=\cdot / \mathrm{\lambda} \mid \text { 米 }\end{array}$ & $\Delta r / \wedge( \pm q / 1 \mu)$ & سن \\
\hline $\begin{array}{c}\mathrm{r}_{\mathrm{s}}=\cdot / 1 \\
\mathrm{p}=\cdot / \cdot \text { 八米 }\end{array}$ & $\begin{array}{l}\mathrm{r}_{\mathrm{s}}=\cdot / \cdot \Delta \\
\mathrm{p}=\cdot / \mu \Delta * * 6\end{array}$ & $\varphi / r V( \pm Y / \varphi)$ & مدت ديمارى \\
\hline $\begin{array}{l}\mathrm{r}_{\mathrm{s}}=-\cdot / / \mathrm{r} \\
\mathrm{p}=\cdot / \cdot \mathrm{r}^{*}\end{array}$ & $\begin{array}{c}r_{s}=\cdot / r r \\
p<\cdot / \cdot \cdot 1 *\end{array}$ & $r / 9 \hookrightarrow( \pm r / r r)$ & دريافت مشاوره جلسات \\
\hline $\begin{array}{l}\mathrm{r}_{\mathrm{p}}=-\cdot / 1 \mathrm{r} \\
\mathrm{p}=\cdot / \cdot \mathrm{r} \text { 米米 }\end{array}$ & $\begin{array}{c}r_{p}=-\cdot / 1 \\
p=\cdot / 11 \text { 米米 }\end{array}$ & $r \Lambda / F F( \pm Y / \Delta S)$ & شاخص توده بدنى \\
\hline- & $\begin{array}{c}r_{p}=\cdot / \wedge r \\
p<\cdot / \cdots \mid \text { 米米 }\end{array}$ & $r \cdot / r r( \pm 1 \cdot / 1 \mu)$ & خود كار آمدى \\
\hline $\begin{array}{c}r_{p}=\cdot / \wedge r \\
p<\cdot / \cdot / \text { 䊉米 }\end{array}$ & - & $\uparrow \wedge / \vee \wedge( \pm 1 \cdot / \varsigma \hookrightarrow)$ & خود مراقبتى \\
\hline
\end{tabular}

جالشى مهم در بيماران مبتلا به بيمارى هاى مزمن، به ويثه بيماران

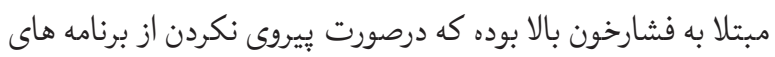

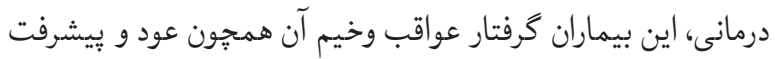

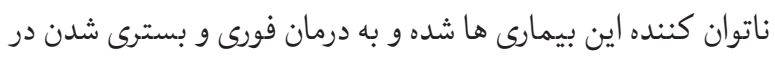

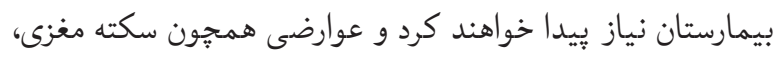
آترواسكلروزيس، سكته قلبى و نارسايى نيز آنان را تهديد مى كنى هند.

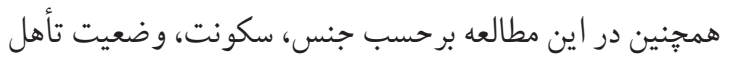
و تعداد فرزند در وضعيت خودمر اقبتى بيماران تفاوتى مشاهده نشد.

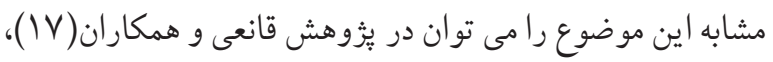

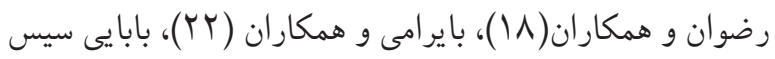

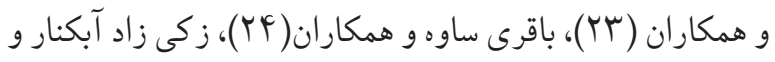

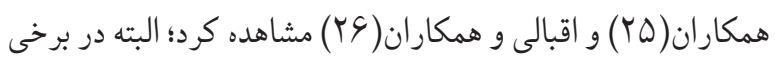
مطالعات نظير مطالعه براتى و همكاران(^) ميزان خودمراقبتى در مدران

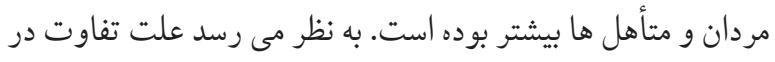

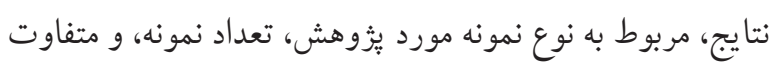

متغيير شاخص توده بدنى وتعداد جلسات دريافت مشاوره، سابقه

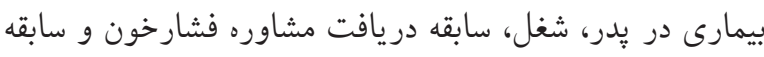
مصرف دارو به عنوان متغيير مداخله كر كنترل شدند.

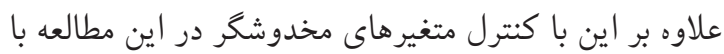
مدل ركرسيون خطى عمومى به ازاى يك واحد افزايش در نمره

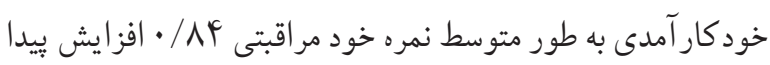

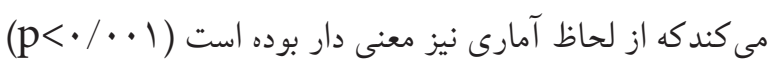

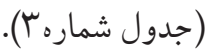
جدول س. نتايج مدل خطى عمومى در تاثير خودكار آمدى بر خودمر اقبتى نمونه هاى مورد بررسى عمدى داتير خودي

\begin{tabular}{|c|c|c|c|c|}
\hline$*$ P-value & $\mathbf{T}$ & خعار & ضريب & خودمراقبتى \\
\hline$<\cdot / \cdot \cdot 1$ & $r \mid / 9 r$ & $\cdot / \cdot r$ & $\cdot / \Lambda F$ & خود كار آمدى \\
\hline.$/ 99$ & $\cdot / \cdot r$ & $\cdot / \cdot 1$ & · & شاخص توده بدنى \\
\hline$\cdot / \mu f$ & . &.$/ 14$ &.$/ 11$ & تعداد جلسات دريافت مشاوره \\
\hline
\end{tabular}


خودمر اقبتى بيماران از نظر شغلى مشاهده نشد. شايد داشتن امنيت شغلى و اقتصادى توان بيماران را درييروى از توصيه هاى كار كنان بهداشتى درمانى و نيز مراقبت از خود افز ايش مى دهد.

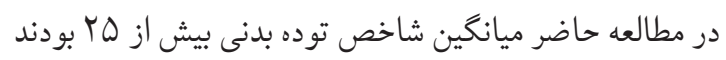
كه با نتايج حاصل از مطالعه ميرزايى علويجه و همكاران( •(1)، Horiy نمايه توده بدنى در مبتلايان به بر فشارخون بالاست، مشابهت داشت.

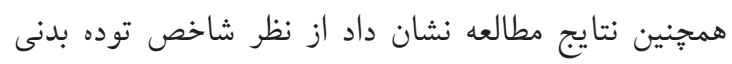

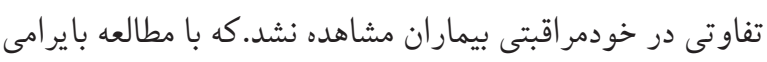

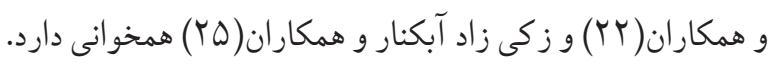

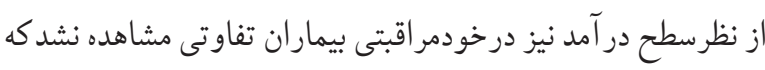

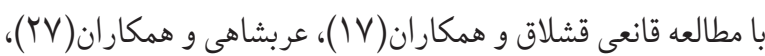

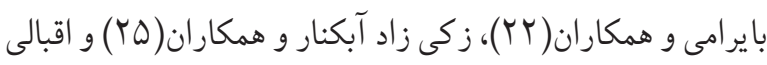

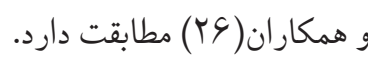
از نظر مدت ابتلا به بيمارى تفاوتى درخودمراقبتى بيماران

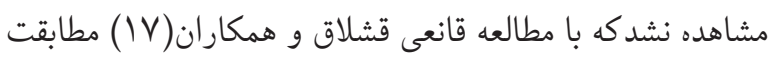

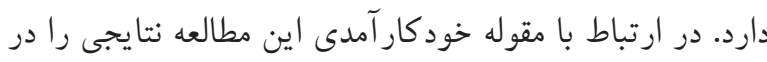
مورد خود كار آمدى رفتارهاى بهداشتى به منظور كنترل فشارخون اردون

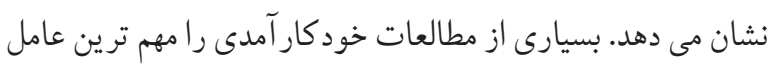

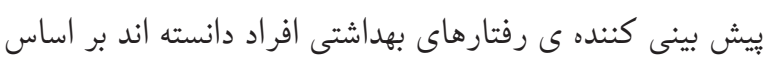
نتايج بدست آمده از اين مطالعه، بيماران مبتلا به فشارخون بالا بـاني از سطح خودكار آمدى مطلوبى در ارتباط با كنترل بيمارى خود

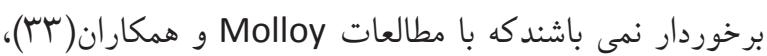

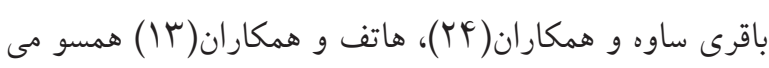

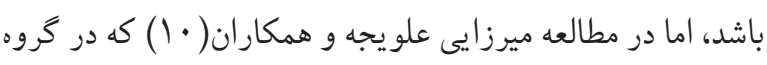

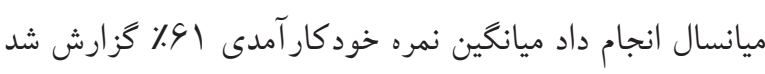

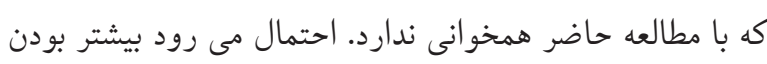

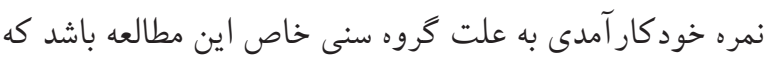
افر اد • مسال به بالامى باشند.

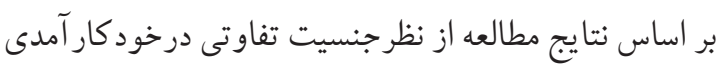

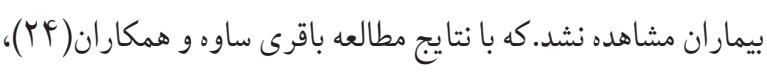

بودن ابزار سنجش خودمراقبتى دانست. در اين مطالعه خودمر اقبتى برحسب ميزان تحصيلات، تفاوت

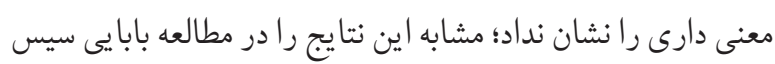

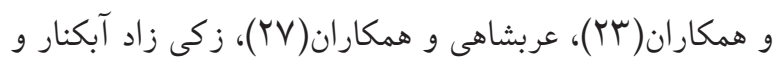

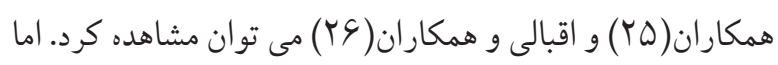

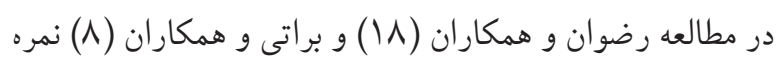

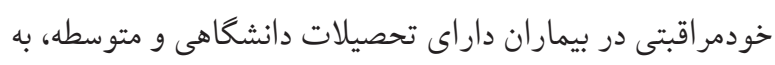
طور معنى دارى بيشتر از ساير گروه ها بود. نتايج نشان داد خودمر اقبتى در بين بيمارانى كه سابقه خانوادكى ني

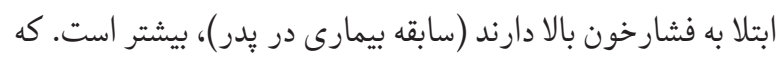

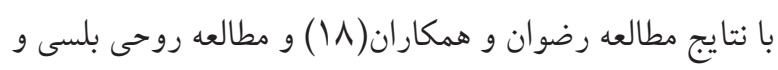

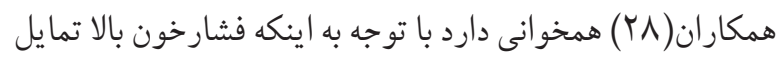

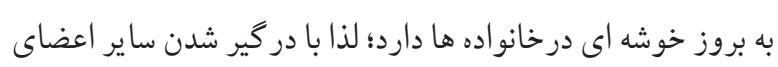

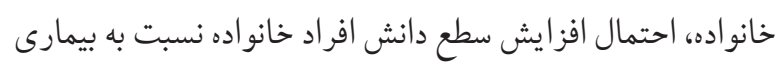
و كنترل آن افزايش مى يابد. همجنين در مطالعه حاضر، بيمارانى كه سابقه دريافت برنامه إنه

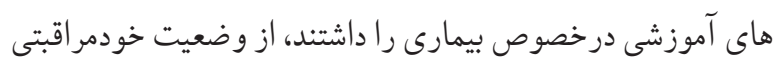

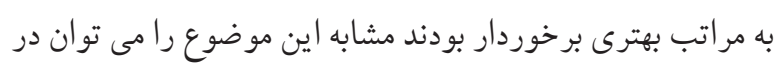
مطالعات رضوان و همكاران(1) )، شاه بداغى و همكاران(

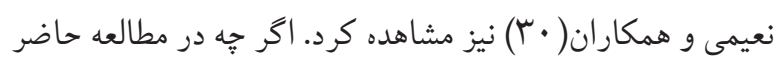

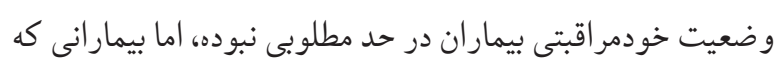

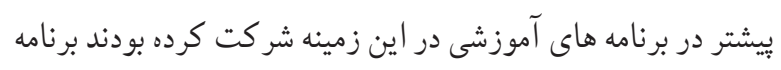

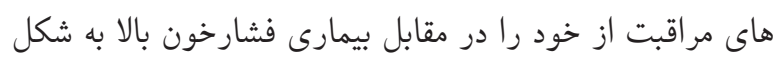

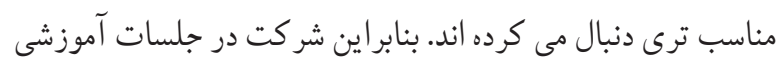

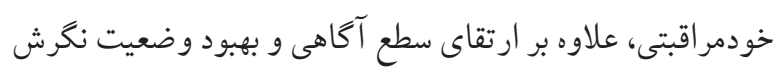

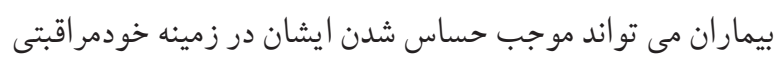

$$
\text { و كنترل عوارض بيمارى شود. }
$$

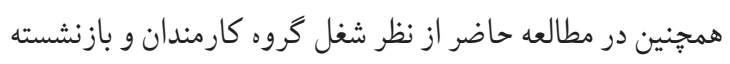

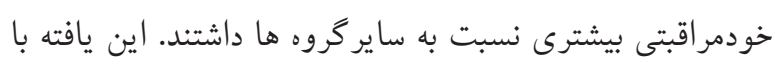

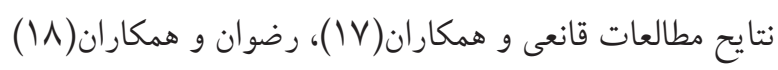

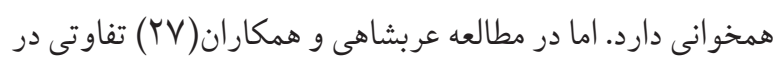


بنابراين خود كار آمدى درمصرف مرتب و به موقع داروهاى ضد فشارخون هر جه بالاتر باشد مبتلايان به فشارخون بهتر مى تواند بيمارى خود را كنترل كند. ميان ميزان درآمد و خودكار آمدى رفتار، رابطه معنادارى در مطالعه

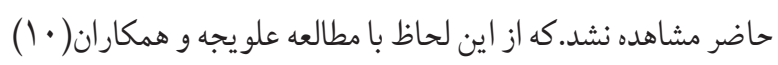

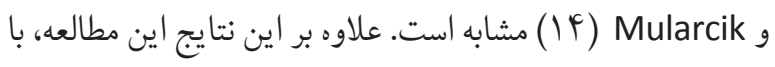

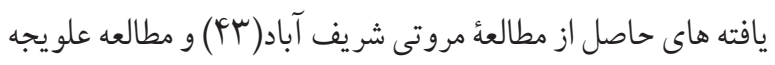

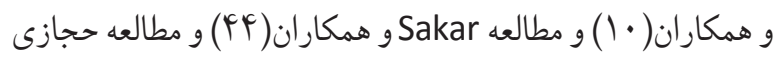

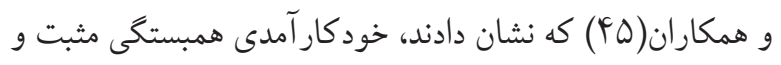
معنى دارى با رفتارهاى خودمر اقبتى دارد همسو است.

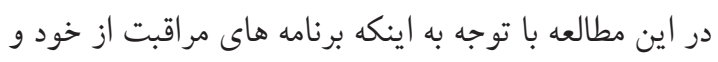

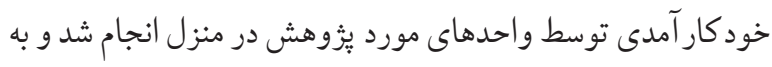

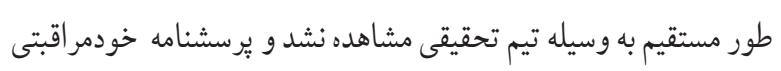

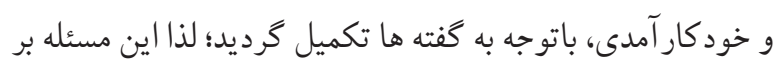

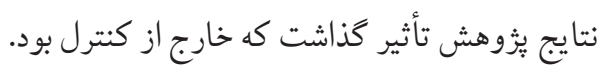
نتيجه كيرى

نتايج مطالعه حاضر نشان داد افراد شركت كننده در اين مطالعه ازخودكارآمدى ضعيفى در ارتباط با رفتارهاى خودمراقبتى برفشارى

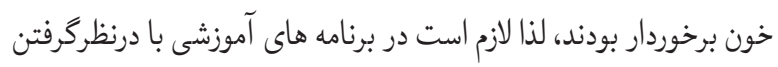

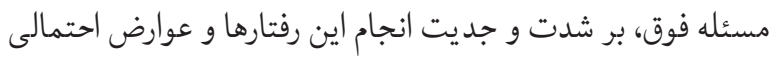

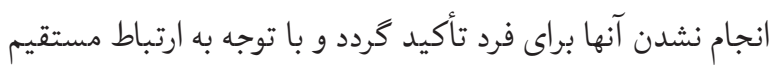

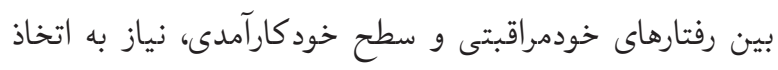

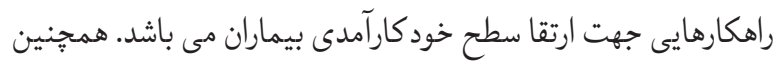
در خصوص فعال و توانمند سازى واحدهاى آموزشى وكادر بهداشتى برانى

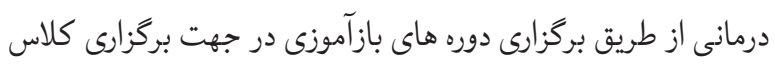
هاى منظم و هدفمند آموزشى براى بيماران توصيه مى كردردي

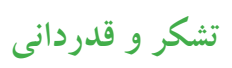

اين يُروهش در قالب طرح تحقيقاتى دانشجويى در مركز يُّوهش دانشجيويان

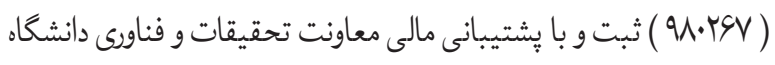

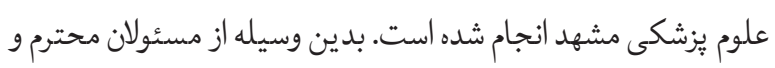
بيماران شركت كننده در طرح تشكر و قدردانى مى كردد.
ميرزايى علو يجه و همكاران( • (1) هم خوانى داشت. اما در مطالعه

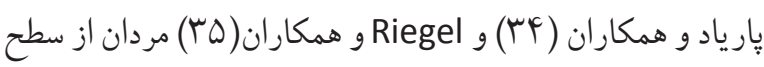

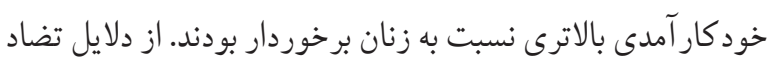

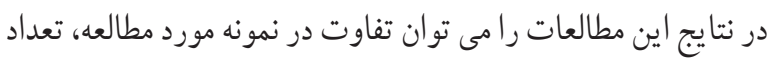

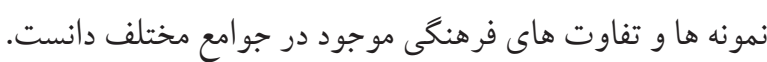

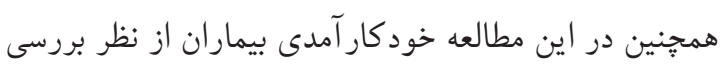

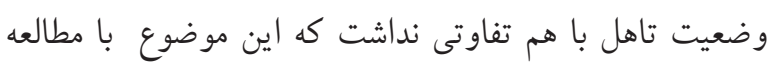

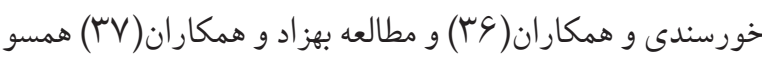

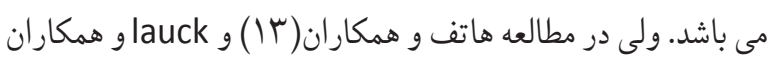

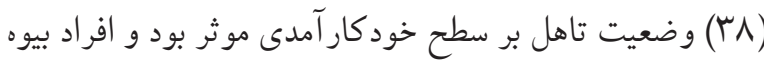

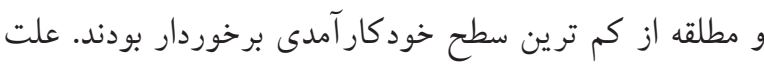

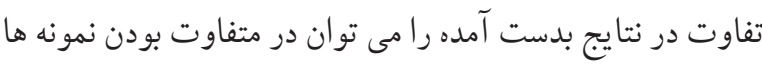

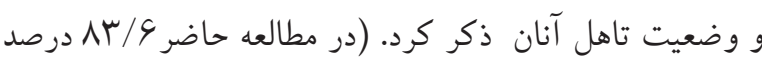

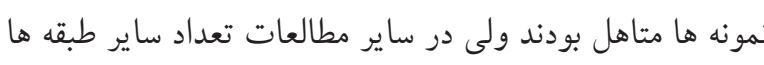
همجيون بيوه و مطلقه نيز قابل توجه بوده است).

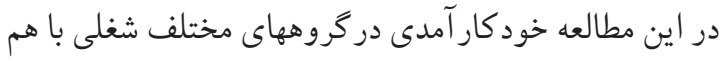
متفاوت بود. كه با مطالعات هاتف و همكاران( (1) برومند و همكاران

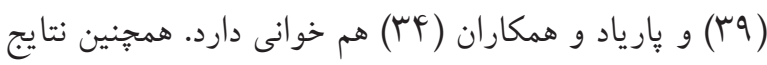

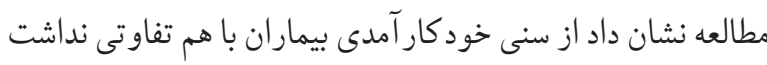

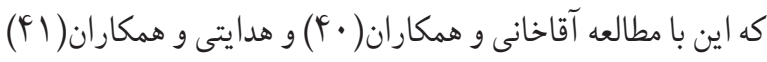

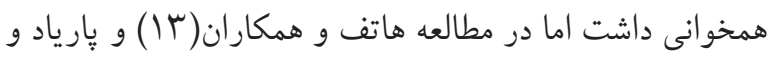
همكاران (TY) بين سن و خود كار آمدى رابطه معنى دار مشاهده

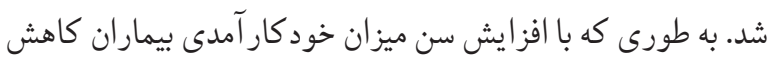

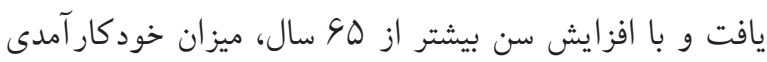
بيماران قلبى كاهش مى يابد. بر اساس نتايج مطالعه بدست آمده بيمارانى كه دارو مصرف

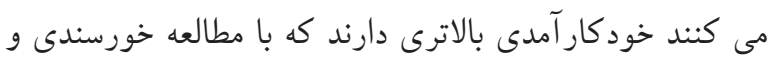

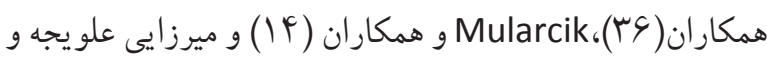

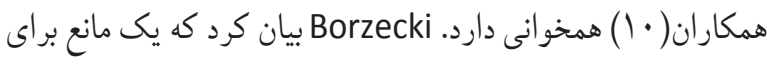

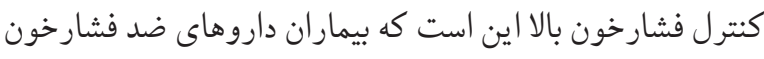

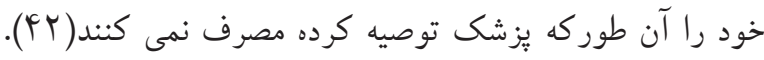




\section{Referenses}

1. SheikhSharafi $H$, Seyedamini B. Assessment of health literacy and self-care in heart failure patients. Journal of Health Literacy. 2017;1(4):203-19. https://doi.org/10.22038/jhl.2017.10854

2. Mohammadpour M, Zarifinezhad E, Armand R. A study of correlation between applied health literacy and self-care behaviors in hypertensive patients of Gachsaran city, 2016 (Iran). Qom University of Medical Sciences Journal. 2018;12(10):55-65. https://doi.org/10.29252/qums.12.10.55

3. Sotodeh Asl N, Neshat Dost H, Kalantery M, Talebi H, Khosravi A. Comparison of the effectiveness of cognitive behavioral therapy and medication on the quality of life in the patients with essential hypertension. Koomesh. 2010;11(4):294-301.

4. Hazavehei S, Dashti S, Moeini B, Faradmal J, Shahrabadi $R$, Yazdi A. Factors related to self-care behaviors in hypertensive individuals based on Health Belief Model. Koomesh. 2015;17(1):37-44.

5. Mansoorian M, Qorbani M, Shafieyan N, Asayesh $H$, Shafieyan Z, Maghsodloo D. Association between life style and hypertension in rural population of Gorgan. Journal of health promotion management. 2012;1(2):23-8.

6. Babaei S, Shakibazadeh E, Shojaeizadeh D, Yaseri M, Mohamadzadeh A. Effectiveness the Theory-Based Intervention Based on Health Belief Model on Health Promotion Lifestyle in Individuals Susceptible to Cardiovascular Diseases. Iranian Journal of Health Education and Health Promotion. 2020;8(3):224-39.. https://doi.org/10.29252/ijhehp.8.3.224

7. Golshiri P, Sadri G, Farajzadegan Z, Sahafi M, Najimi A. Is there any association between family function self care in women? Journal of Isfahan Medical School. 2012;29(166):1-9.

8. Barati M, Darabi D, Moghimbeigi A, Afsar A. Self-regulation behaviors of hypertension and related factors among hypertensive patients. Journal of Fasa University of Medical Sciences. 2011;1(3):116-22.

9. Gangi S, Peyman N, Esmaily H. Comparative study of two methods of fluid therapy with Ringer's Lactate and Ringer's solution. medical journal of mashhad university of medical sciences. 2018;60(6):792-803.

10. Mirzaei Alavijeh M, Nasirzadeh M, Jalilian F, Mostafavei F, Hafezi M. Self-efficacy of health promotion behaviors in hypertensive patients. Daneshvar. 2012;19(1):51-8..

11. Vrijhoef $H$, Diederiks J, Spreeuwenberg C, Wolffenbuttel B, Van Wilderen L. The nurse specialist as main careprovider for patients with type 2 diabetes in a primary care setting: effects on patient outcomes. International journal of nursing studies. 2002;39(4):441-51.
https://doi.org/10.1016/S0020-7489(01)00046-3

12. Warren-Findlow J, Seymour RB, Huber LRB. The association between self-efficacy and hypertension self-care activities among African American adults. Journal of community health. 2012;37(1):15-24. https://doi.org/10.1007/s10900-011-9410-6 PMid:21547409 PMCid:PMC3179559

13. Hatef M, Sharif Nia H, Mousavinasab N, Esmaeili R, Shafipour V. Self-efficacy and prediction of associated factors in patients with chronic diseases. Journal of Mazandaran University of Medical Sciences. 2018;28(162):86-94.

14. Mularcik KA. Self-efficacy toward health behaviors to improve blood pressure in patients who receive care in a primary care network: Electronic Thesis or Dissertation. Ohio State University, 2010. https://etd.ohiolink.edu/.

15. Ghanei Gheshlagh r (Ph.D)1 PnPD, Ghalenoee m(M.Sc)3, Dalvand s (Ph.D student)4, Farajzadeh m (M.Sc) 5, Ebadi a (Ph.D)*6 Psychometric properties of Persian version of Hypertension Self-Care Profile in patients with high blood pressure 2018;1(21):25-32.

16. Gheshlagh RG, Parizad N, Ghalenoee M, Dalvand S, Baghi V, Najafi F, et al. Psychometric features of the persian version of self-efficacy tool for patients with hypertension. International Cardiovascular Research Journal. 2018;12(2):50-6.

17. Ghanei Gheshlagh R, Parizad N, Ghalenoee M, Dalvand S, Farajzadeh M, Ebadi A. Psychometric properties of Persian version of Hypertension Self-Care Profile in patients with high blood pressure. Koomesh. 2019;21(1):25-32.

18. Rezvan S, Besharati M, Khodadadpoor M, Matlabi M, Fathi A, Salimi A, et al. Self-Care Assessment of Patients with Hypertension in Qom City in 2016 (Iran). Qom University of Medical Sciences Journal. 2018;12(4):72-80. https://doi.org/10.29252/qums.12.4.72

19. Newell M, Modeste N, Marshak HH, Wilson C. Health beliefs and the prevention of hypertension in a black population living in London. Ethnicity \& disease. 2009;19(1):35.

20. Delavari A, Horri N, Alikhani S, Gouya M, Mahdavi A, Hosseini $S$, et al. Prevalence of hypertension in Iranian urban and rural populations aged over 20 years in 2004 . Journal of Mazandaran University of Medical Sciences. 2007;17(58):79-86.

21. Fuladvandi $M$, Safarpour $H$, Malekyan L, Moayedi $S$, Mahani MA, Salimi E. The Survey of self-regulation behaviors and related factors in elderly with hypertension in south-east of Iran. Health. 2017;9(04):592. https://doi.org/10.4236/health.2017.94042

22. Bairami S, Fathi $Y$, Mohammadinasab S, Barati $M$, Mohammadi Y. Relationship between self-care behaviors and quality of life among hypertensive patients visiting 
comprehensive health centers in Hamadan, Iran. Journal of Education and Community Health. 2017;4(1):20-7. https://doi.org/10.21859/jech.4.1.20

23. $\mathrm{k}$ bsmrsmhbztmfm. The effect of lifestyle modification educational intervention on blood pressure control in patients with hypertension. Journal of Education and Community Health. 2016;3(1):12-9. https://doi.org/10.21859/jech-03012

24. saveh Mi B, Ashk Torab T. Borz Abadi Farahani Z, Zayeri F, Zohari Anboohi S. The relationship between self-efficacy and self-care behaviors in J Shahid Beheshti School Nurs Midwif. 2012;22(78):4715.

25. Abkenar MZ, Ghofranipour F, Kashi Z, Montazeri A. Effect of the Islamic Self-care Nutrition Method on the Lipid Profile of Patients with Type 2 Diabetes: A Randomized Clinical Controlled Trial. Open Access Macedonian Journal of Medical Sciences. 2020;8(B):161-7. https://doi.org/10.3889/oamjms.2020.4217

26. Eghbali T, Salehi S. The Effect of Nursing Intervention Education on Self-care Behaviors in Patients with High Blood Pressure Referred to Healthcare Centers in Kermanshah Province. Iranian Journal of Rehabilitation Research. 2017;4(1):32-7.

27. Arabshahi A, Gharlipour Z, Mohammadbeigi A, Mohebi S. The Effect of Education Based on Spousal Social Support on Improving Self-Care Behaviors in Men with High Blood Pressure. Qom University of Medical Sciences Journal. 2020;14(2):34-46. https://doi.org/10.29252/qums.14.2.34

28. Balasi L, Paryad E, Kazemnezhad Leili E, Booraki S, SadeghiMeibodi A, Nassiri Sheikhani N. Study status of care adherence and its related factors in patients undergoing Coronary Artery By pass Surgery. Journal of Holistic Nursing And Midwifery. 2015;15(25):3.

29. Shahbodaghi Z, Borhani F, Rayyani M. The effects of salfcare program on blood pressure of patients with diabetes. Medical - Surgical Nursing Journal. 2014;3(3):163-169.

30. Naeimi E, Malekzadeh J, Hadinia A, Sharifi B, Mosavizadeh A. Assessment of Knowledge and Practice of Hypertension Patients in Boyer Ahmad City in 2008. Armaghane danesh. 2011;16(5):302-9.

31. Hori $Y$, Toyoshima H, Kondo T, Tamakoshi K, Yatsuya H, Zhu $S$, et al. Gender and age differences in lifestyle factors related to hypertension in middle-aged civil service employees. Journal of epidemiology. 2003;13(1):38-47. h tt p s://doi . org/10.2188/jea.13.38 PMid:12587612

32. Ohira T, Iso H, Tanigawa T, Sankai T, Imano H, Kiyama M, et al. The relation of anger expression with blood pressure levels and hypertension in rural and urban Japanese communities. Journal of hypertension. 2002;20(1):21-7. https://doi.org/10.1097/00004872-200201000-00005
PMid:11791022

33. Molloy GJ, Randall G, Wikman A, Perkins-Porras L, MesserliBürgy N, Steptoe A. Type D personality, self-efficacy, and medication adherence following an acute coronary syndrome. Psychosomatic medicine. 2012;74(1):100-6. https://doi.org/10.1097/PSY.0b013e31823a5b2f PMid:22155940

34. Paryad E, Hosseinzade T, Kazemnejad E, Asiri S. A study of self-efficacy in patients with coronary artery disease and its predictors. Qom Univ Med Sci J. 2013;7(2):41-8.

35. Riegel B, Dickson VV, Kuhn L, Page K, Worrall-Carter L. Gender-specific barriers and facilitators to heart failure self-care: a mixed methods study. International journal of nursing studies. 2010;47(7):888-95. https://doi.org/10.1016/j.ijnurstu.2009.12.011 PMid:20079902

36. Khorsandi M, Fekrizadeh Z, Roozbahani N. Investigation of the effect of education based on the health belief model on the adoption of hypertension-controlling behaviors in the elderly. Clinical interventions in aging. 2017;12:233. h tt p s: / / d o i.org / 10.2147 / CIA.S 117142 PMid:28184154 PMCid:PMC5291452

37. Behzad Y, Hagani H, Bastani F. Effect of empowerment program with the telephone follow-up (tele-nursing) on self-efficacy in self-care behaviors in hypertensive older adults. The Journal of Urmia Nursing and Midwifery Faculty. 2016;13(11):1004-15.

38. LauckS, JohnsonJL, RatnerPA.Self-carebehaviourandfactors associated with patient outcomes following same-day discharge percutaneous coronary intervention. European Journal of Cardiovascular Nursing. 2009;8(3):190-9. https://doi.org/10.1016/j.ejcnurse.2008.12.002 PMid:19162553

39. Boroumand S, Shahriari M, Abbasi Jebeli M, Baghersad Z, Baradaranfard F, Ahmadpoori F. Determine the level of self-efficacy and its related factors in patients with ischemic heart disease: A descriptive correlational study. Iranian Journal of Nursing Research. 2015;9(4):61-9.

40. Aghakhani N, Alinejad V, Khademvatan K, Golmohamadi F. The Effect Of Self-Care Education On The Self Efficacy In Myocardial Infarction Hospitalized Patients In Seyeid Al-Shohada Educational \& Treatment Center, Urmia, 2017. The J Urmia Nurs Midwifery Fac. 2018;16(6):412-22.

41. Hedayati B. Effect of Family-Based Empowerment Model on the Self-Efficacy of Hypertensive Elderly People. Iranian Journal of Ageing. 2018;13(1):86-97. https://doi.org/10.21859/sija.13.1.86

42. Borzecki AM, Oliveria SA, Berlowitz DR. Barriers to hypertension control. The American heart journal. 2005;149(5):785-94. https://doi.org/10.1016/j.ahj.2005.01.047 PMid:15894958 
43. Morowatisharifabad M, Rouhani Tonekaboni N. Perceived self-efficacy in self-care behaviors among diabetic patients referring to Yazd Diabetes Research Center. Journal of Birjand University of Medical Sciences. 2008;15(4):91-9.

44. Sarkar U, Fisher L, Schillinger D. Is self-efficacy associated with diabetes self-management across race/ethnicity and health literacy? Diabetes care. 2006;29(4):823-9. https://doi.org/10.2337/diacare.29.04.06.dc05-1615 PMid:16567822
45. Hejazi S, Peyman N, Tajfard M, Esmaily H. The impact of education based on self-efficacy theory on health literacy, self-efficacy and self-care behaviors in patients with type 2 diabetes. Iranian Journal of Health Education and Health Promotion. 2017;5(4):296-303. https://doi.org/10.30699/acadpub.ijhehp.5.4.296 